\section{IN THE NEWS}

Ebola vaccine trials Human trials testing the safety of an Ebola virus vaccine were recently begun by researchers from the Vaccine Research Centre (VRC) at the National Institute of Allergy and Infectious Diseases (NIAID).

Ebola virus is highly infectious with a fatality rate of up to $90 \%$. Although initially rare, the frequency of Ebola virus epidemics has increased in recent years. According to a recent report from the World Health Organization, an ongoing Ebola-virus outbreak in the Congo has so far killed 18 people and this, as Gary Nabel (VRC Director) said, "provides a stark reminder of the need to rapidly develop vaccines against such perilous infections" (Reuters). The deadly characteristics of Ebola virus have also led US experts to suggest that it could be used as a biological weapon. So, as Anthony Fauci (NIAID Director) said, "an effective Ebola vaccine not only would provide a lifesaving advance in countries where the disease occurs naturally, it would also provide a medical tool to discourage the use of Ebola virus as an agent of bioterrorism" (BBC).

The vaccine used in the trials - a DNA vaccine made by a San Diego-based biotechnology company, Vical Inc. - was one component of a vaccine shown to protect monkeys from subsequent infection with Ebola virus. The vaccine is synthesized from modified, inactivated Ebolavirus genes and according to Barney Graham, the director of the clinical trials, "it employs new technology known to safely stimulate broad immune responses". It is hoped that the vaccine could be used as the first part of a prime-boost vaccination protocol and Gary Nabel has said that "expanded human trials of Ebola vaccines using the prime-boost strategy could begin by 2005" (NIH News)

Karen Honey

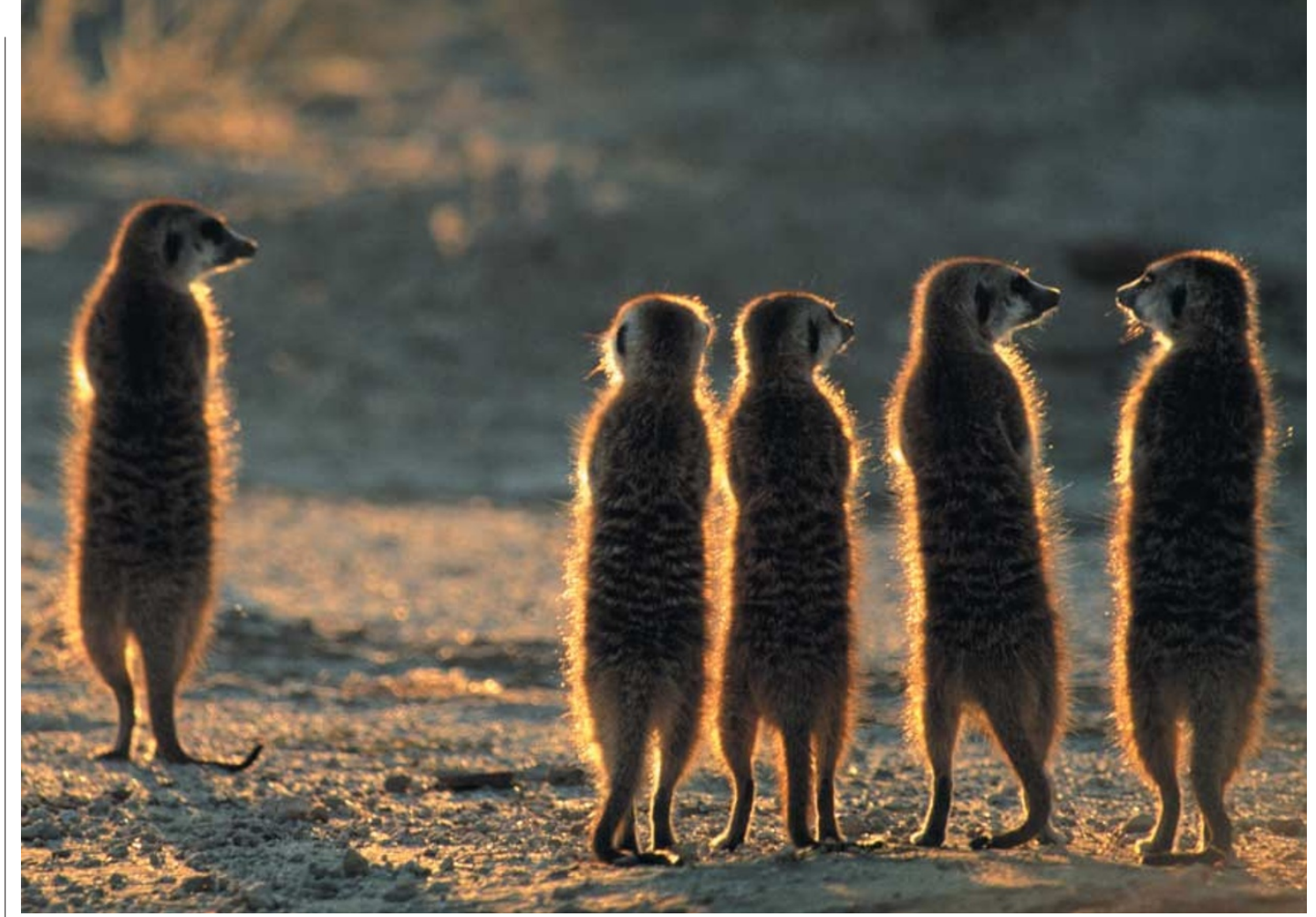

LYMPHOCYTE DEVELOPMENT

\title{
Shared survival signals
}

A fine balance between life and death ensures that appropriate numbers of $\mathrm{T}$ and $\mathrm{B}$ cells are produced and maintained in the periphery. One regulator of this balance is the cytokine interleukin-7 (IL-7), which promotes the survival of lymphocytes. Stanley Korsmeyer and colleagues have now identified the BCL-2-family member myeloid-cell leukaemia 1 (MCL1) as a crucial component of the signalling pathway downstream of IL-7.

Mcl1 deficiency is embryonic lethal, so these studies involved a conditional deletion strategy (Cre-lox $P$ ). Mice with a conditional T-lineage deletion of Mcl1 had decreased numbers of peripheral $\mathrm{CD} 4^{+}$and $\mathrm{CD}^{+} \mathrm{T}$ cells. Similarly, B-lineage deletion markedly reduced the number of splenic and lymph-node B cells. The authors then looked at the specific developmental stage that is blocked in each case. T-lineage Mcl1-deficient mice had a reduced number of single-positive and double-positive thymocytes. The block was shown to occur earlier in the double-negative (DN) subset at the DN2 to DN3 transition, and the DN2 population had an increased number of apoptotic cells. For Mcl1 deficiency in the B-cell lineage, both pro-B- and pre-B-cell populations were reduced in the bone marrow, and increased apoptosis was observed in the pro-B-cell subset.

Interestingly, both pro-B-cell and $\mathrm{DN} 2$ populations are known to require cytokine survival signals and the developmental blocks observed in the Mcl1-deficient mice are similar to those seen for IL-7- or IL-7 receptor-deficient mice. Moreover, in vitro culture of DN1 and DN2 thymocytes with IL-7 resulted in an increase in Mcl1 messenger RNA and protein levels. So, what is the role of MCL1 in IL-7 signalling? Of the two pro-apoptotic $\mathrm{BH} 3$-only BCL-2-family proteins (BIM and BAD) that are affected by cytokinedependent survival pathways, MCL1 interacted strongly with BIM. Therefore, MCL1 might function to prevent apoptosis of developing lymphocytes by binding BIM and preventing BIM-mediated activation of the pro-apoptotic proteins BAX and BAK.

This developmental model was confirmed for the maintenance of peripheral lymphocytes. The total deficiency of Mcl1 in adult mice led to depletion of $\mathrm{T}$ and $\mathrm{B}$ cells from the spleen, and adoptive-transfer experiments showed that the requirement for Mcl1 is intrinsic to $\mathrm{T}$ and $\mathrm{B}$ cells. In vitro, the IL-7-mediated survival of mature $\mathrm{T}$ cells required Mcl1. So, these studies have indicated conservation of cytokinedependent survival pathways for the development and maintenance of both $\mathrm{T}$ and $\mathrm{B}$ cells.

Kirsty Minton

\section{(2) References and links}

ORIGINAL RESEARCH PAPER Opferman, J. T. et al. Development and maintenance of $\mathrm{B}$ and $\mathrm{T}$ lymphocytes requires anti-apoptotic MCL-1. Nature 426, 671-676 (2003)

WEB SITE

Stanley Korsmeyer's lab: http://research.dfci.harvard.edu/korsmeyer/ 